\title{
Características de fruto y semilla de chile huacle (Capsicum annuum L.) producido en hidroponia
}

\author{
Fruit and seed characteristics of chili huacle \\ (Capsicum annuum L.) produced in hydroponics \\ Jair San Juan Martínez ${ }^{1}$, Teodulfo Aquino-Bolaños ${ }^{1}$, \\ Yolanda Donaji Ortiz-Hernández ${ }^{1}$, Serafín Cruz-Izquierdo ${ }^{2}$
}

\section{RESUMEN}

El chile huacle (Capsicum annuиm L.) es una hortaliza endémica de Oaxaca, México con bajo rendimiento. El objetivo de este estudio fue evaluar tres concentraciones de solución Steiner: 10, 15 y 20 meq L ${ }^{-1}$ en las características morfométricas de frutos y en la calidad de las semillas del chile huacle, parámetros importantes para la producción y conservación de las especies. El experimento se llevó a cabo en macrotúnel con cubierta de polietileno y malla sombra al 30\%. Se utilizó un diseño completamente al azar con 50 repeticiones por tratamiento, la unidad experimental fue una planta por bolsa de polietileno de $20 \mathrm{~L}$ con sustrato de agrolita®. Las variables evaluadas en fruto fueron: peso seco, largo, ancho, grosor de pericarpio. En semillas: peso y número de semillas, área, largo, ancho, peso de 1000 semillas, contenido de humedad, viabilidad, porcentaje de germinación y envejecimiento acelerado. Las características en el fruto y semillas del chile huacle fueron estadísticamente mayores $(\mathrm{P} \leq 0,05)$ con la aplicación a las plantas de la solución Steiner de $20 \mathrm{meq} \mathrm{L}^{-1}$. Se produjeron frutos con mayor peso seco $(5,56 \mathrm{~g})$, largo $(7,1 \mathrm{~cm}) \mathrm{y}$ ancho $(4,21 \mathrm{~cm})$. Asimismo, las semillas fueron de mayor calidad física y fisiológica, mostrando un área de 15,9 mm², largo de 4,9 mm, $6,19 \mathrm{~g}$ en el peso de 1,000 semillas, plántulas con la radícula de mayor tamaño $(5,02 \mathrm{~cm}), 96 \%$ de viabilidad y germinación. En los tres tratamientos, el envejecimiento acelerado de las semillas fue del $80 \%$ de germinación, característica deseable para la reproducción y conservación de la especie.

Palabras clave: envejecimiento acelerado, germinación, viabilidad de la semilla, soluciones nutritivas, recurso fitogenético.

\section{ABSTRACT}

The Huacle chili (Capsicum annuum L.) is an endemic vegetable from Oaxaca, Mexico with low yield. The objective of this study was to evaluate three concentrations of Steiner solution: 10, 15 and 20 meq $L^{-1}$ in the morphometric characteristics of fruits and in the quality of Huacle chili seeds, important parameters for the production and conservation of the species. The experiment was carried out in macro tunnel with polyethylene cover and $30 \%$ shade mesh. A completely random design with 50 repetitions per treatment was used, the experimental unit was one plant per 20 L polyethylene bag with agrolita ${ }^{\circledR}$ substrate. The variables evaluated in fruit: dry weight, length, width, thickness of pericarp. In seeds: weight and number of seeds, area, length, width, weight of 1,000 seeds, moisture content, viability, percentage of germination and accelerated aging. The characteristics in the fruit and seeds of the Huacle chili were statistically higher $(P \leq 0,05)$ with the application to the plants of the Steiner solution of $20 \mathrm{meq} \mathrm{L}^{-1}$. Fruits with higher dry weight $(5,56 \mathrm{~g})$, length $(7,1 \mathrm{~cm})$ and width $(4,21 \mathrm{~cm})$. Likewise, the seeds were of greater physical and physiological quality, showing an area of 15,9 $\mathrm{mm}^{2}$, length of $4,9 \mathrm{~mm}, 6,19 \mathrm{~g}$ in the weight of 1,000 seeds, seedlings with the largest radicle $(5,02 \mathrm{~cm}), 96 \%$ viability and germination. In all three treatments, the accelerated aging of the seeds was $80 \%$ germination, a desirable characteristic for the reproduction and conservation of the species.

Key words: accelerated aging, germination, seed viability, nutritive solutions, phytogenetic resource.

1 Instituto Politécnico Nacional. Centro Interdisciplinario de Investigación para el Desarrollo Integral Regional Unidad Oaxaca (CIIDIR - Oaxaca). Hornos 1003. C.P. 71230, Santa Cruz Xoxocotlán, Oaxaca, Oaxaca, México.

2 Colegio de Postgraduados. Campus Montecillo. Recursos Genéticos y Productividad - Fisiología Vegetal. Montecillo, Estado de México. México.

* Autor para correspondencia/Corresponding author: yortiz@ipn.mx

Fecha de Recepción: 02 Marzo, 2019.

Fecha de Aceptación: 07 Abril, 2019. 


\section{Introducción}

El chile huacle o chilhuacle (Capsicum annuиm L.), es endémico y cultivado en la región de la Cañada de Oaxaca, México, presenta variantes en el color de fruto: negro, amarillo y rojo (LópezLópez et al., 2016). En el año 2015 se registraron 10 ha cultivadas a cielo abierto bajo un sistema de riego rodado con un rendimiento promedio menor a una tonelada por hectárea de chile deshidratado (López-López et al., 2016).

La semilla que emplean los productores para la producción de chile huacle, procede de frutos cosechados en el ciclo inmediato anterior y la selección de los mismos se realiza posterior al proceso del deshidratado, para ello eligen los mejores frutos en cuanto a forma, tamaño y color, pero en ningún caso se efectúa alguna estrategia para incrementar su calidad.

La producción del genero Capsicum bajo condiciones de agricultura protegida y sistemas hidropónicos puede ser una alternativa viable (Tapia-Vargas et al., 2016). La concentración de la solución nutritiva es un factor que influye en el número y tamaño de los frutos en chile de agua (Valentín-Miguel et al., 2013) y chile habanero (Alejo-Santiago et al., 2015).

Por otra parte, la deficiencia de Nitrógeno $(\mathrm{N})$, Fosforo (P), Potasio (K), Calcio (Ca), Magnesio (Mg), Azufre (S), Boro (B), Hierro (Fe) y Zinc ( $\mathrm{Zn}$ ) en el género Capsicum puede reducir la floración, la fructificación y el tamaño de los frutos (Ramalho do Rêgo et al., 2016). Las fuentes de nutrimentos para las plantas tienen efecto en el tamaño y peso de los frutos de chile (Valadez Sánchez et al., 2016). La deficiencia de $\mathrm{P}$ y $\mathrm{K}$ en los cultivos de hortalizas ocasionan poco desarrollo de semillas y semillas vanas (Izquierdo y Granados Ortiz, 2011).

La semilla juega un papel fundamental en la reproducción y en la determinación del valor económico de las diferentes especies al igual que en su conservación, porque las semillas de alta calidad conservan su poder germinativo y resisten condiciones adversas de almacenamiento (AyalaVillegas et al., 2014). La calidad de una semilla está determinada por sus propiedades físicas y fisiológicas como lo son: peso, contenido de humedad, viabilidad, germinación y vigor (ISTA, 2012). La viabilidad es un indicador del potencial metabólico de la semilla; la cual se evalúa durante las primeras horas después de la hidratación estimando indirectamente el potencial germinativo (Copeland y McDonald, 2001).

Basado en los antecedentes, en esta investigación se tuvo como objetivo comparar tres concentraciones de solución Steiner en las características morfométricas de frutos y en la calidad de semillas del chile huacle (Capsicum annuиm L.).

\section{Materiales y métodos}

La investigación se realizó de enero a junio de 2018 bajo macrotúnel de $240 \mathrm{~m}^{2}$ con cubierta de polietileno y malla sombra al $30 \%$ en los Valles

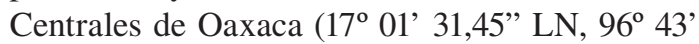
12,7 " LO) a $1526 \mathrm{msnm}$. La siembra del almácigo se realizó el $1^{\circ}$ de enero de 2018 en charolas de poliestireno de 200 cavidades utilizando como sustrato turba Sphagnum $\AA$ y agrolita $\AA$ en proporción 2:1. Cada 24 h se aplicó un riego con solución Steiner al 25\%. Cuando las plántulas alcanzaron en promedio $15 \mathrm{~cm}$ de altura y de tres a cinco hojas verdaderas, se realizó el trasplante colocando una planta por bolsa de polietileno de $20 \mathrm{~L}$ de capacidad utilizando como sustrato Agrolita ${ }^{\circledR}$.

Los tratamientos evaluados fueron tres concentraciones: 20, 15 y 10 en meq $\mathrm{L}^{-1}$ de solución Steiner (Tabla 1). Las fuentes de fertilizantes fueron: nitrato de calcio $\mathrm{Ca}\left(\mathrm{NO}_{3}\right)_{2}$, nitrato de potasio $\mathrm{KNO}_{3}$, sulfato de magnesio $\mathrm{MgSO}_{4}$ y ácido fosfórico $\mathrm{H}_{3} \mathrm{PO}_{4}$

Se utilizó un diseño completamente al azar con 50 repeticiones por tratamiento, la unidad experimental fue una planta de chile huacle por bolsa, que se distribuyeron en filas de $1,2 \mathrm{~m}$ de ancho por 0,6 m de separación entre bolsas. Se tomaron al azar 10 plantas de cada tratamiento para obtener 50 frutos fisiológicamente maduros de cada tratamiento, donde se evaluaron las variables: peso seco, largo, ancho, grosor de pericarpio y número de semillas. Se consideró como parámetro de fruto

Tabla 1. Tratamientos aplicados a chile huacle bajo macrotúnel.

\begin{tabular}{|c|c|c|c|c|c|c|}
\hline \multirow{2}{*}{$\begin{array}{l}\text { Tratamiento } \\
(\text { meq L-1) }\end{array}$} & \multicolumn{3}{|c|}{ Cationes (meq L-1) } & \multicolumn{3}{|c|}{ Aniones $\left(\mathrm{meq} \mathrm{L}^{-1}\right)$} \\
\hline & $\mathrm{K}^{+}$ & $\mathrm{Ca}^{2+}$ & $\mathrm{Mg}^{2+}$ & $\mathrm{NO}_{3}^{-}$ & $\mathrm{H}_{3} \mathrm{PO}_{4}^{-}$ & $\mathrm{SO}^{2-}{ }_{4}$ \\
\hline 20 & 7 & 9 & 4 & 12 & 1 & 7 \\
\hline 15 & 5,25 & 6,75 & 3 & 9 & 0,75 & 5,25 \\
\hline 10 & 3,5 & 4,5 & 2 & 6 & 0,5 & 3,5 \\
\hline
\end{tabular}

$\mathrm{K}^{+}=$potasio, $\mathrm{Ca}^{2+}=$ calcio, $\mathrm{Mg}^{2+}=$ magnesio, $\mathrm{NO}_{3}{ }^{-}=$nitratos, $\mathrm{H}_{3} \mathrm{PO}_{4}^{-}=$ácido fosfórico, $\mathrm{SO}_{4}{ }^{2-}=$ sulfatos . 
fisiológicamente maduro cuando se presentó de un 80 a 100\% en el fruto el cambio de color de verde a café (Figura 1A y 1B).

El peso seco se obtuvo con una balanza analítica (Ohaus Plus mod. AP310, USA). Posterior a la cosecha los frutos fueron secados en una estufa (Cole-Parmer mod. 05015_58, USA), a $70^{\circ} \mathrm{C}$ durante $72 \mathrm{~h}$. El largo, ancho y grosor de pericarpio (Figura 1C) se midió con un vernier digital $\pm 0,01 \mathrm{~mm}$ (MITUTOYO mod. 500-196-30, JPN).

Asimismo, se contaron ocho repeticiones de 100 semillas con morfología normal y testa entera de cada tratamiento y se pesaron en una balanza analítica (Ohaus Plus AP310, USA) con precisión de 0,0001 g. Para cada tratamiento se calculó: media, varianza, desviación estándar y coeficiente de variación, este último fue menor del $4 \%$.

El contenido de humedad de las semillas se determinó en dos muestras aleatorias de $2 \mathrm{~g}$ de cada tratamiento, las cuales fueron expuestas en una estufa (Cole-Parmer mod. 05015_58, USA) a $103{ }^{\circ} \mathrm{C}$ durante $17 \pm 1 \mathrm{~h}$. Se registró el peso fresco y posterior al secado se obtuvo el peso seco, y se calculó el contenido de humedad (ISTA, 2012).

La viabilidad de las semillas se realizó mediante la prueba de cloruro de tetrazolio, se utilizaron 100 semillas de cada tratamiento, las cuales fueron embebidas en agua destilada durante $12 \mathrm{~h}$, posteriormente se realizó un corte longitudinal y fueron sumergidas en solución de cloruro de 2,3,5-trifeniltetrazolio (1\%, $\mathrm{p} / \mathrm{v})$.

La prueba de germinación para cada tratamiento se hizo con cuatro repeticiones de 25 semillas, que se colocaron sobre toallas de papel húmedas (Sanitas ${ }^{\circledR}$, Kimberly Clark) en cajas de plástico transparente bajo cámara de germinación SD8900
(Seedburo, USA) a $25 \pm 1^{\circ} \mathrm{C}$ con luz constante durante 14 días. Posteriormente, se realizó el conteo de semillas germinadas para obtener el porcentaje de germinación y aleatoriamente en 20 plántulas se midió la longitud de plúmula y radícula con un escalímetro Kaiser K-01.

El envejecimiento acelerado se realizó con 100 semillas de cada tratamiento sometidas a $45 \pm 1{ }^{\circ} \mathrm{C}$ y humedad relativa del $100 \%$, por un periodo de $72 \mathrm{~h}$ (ISTA, 2012). Posteriormente fueron germinadas y se calculó el porcentaje de germinación y longitudes de plúmula y radícula como se describió anteriormente para las semillas no sometidas a envejecimiento.

Los análisis de varianza y comparación de medias con la prueba de Tukey $(\mathrm{P} \leq 0,5)$ fueron analizados para cada variable mediante el programa $S A S^{\circledR}$ versión 9,0 (SAS, 2002). Las asociaciones entre variables se realizaron mediante un análisis de correlación $(\mathrm{P} \leq 0,01$ y 0,05$)$ con este mismo programa. Anterior a los análisis los porcentajes de germinación fueron transformados mediante la función arcoseno.

\section{Resultados y discusión}

\section{Características físicas del fruto}

Los datos obtenidos mostraron diferencias significativas $(\mathrm{P} \leq 0,05)$, con 20 meq $\mathrm{L}^{-1}$ respecto a las concentraciones de 15 y $10 \mathrm{meq} \mathrm{L}^{-1}$ se tuvo mayor respuesta para las variables: peso seco, largo y ancho de fruto (Tabla 2), similar a lo encontrado en chile serrano (Cruz-Crespo et al., 2014), quienes observaron que a mayor concentración de la solución nutritiva fue mayor la acumulación de materia seca y el tamaño de fruto, similar también a lo reportado por Bosland y Votava (2012) para otros tipos de chiles.

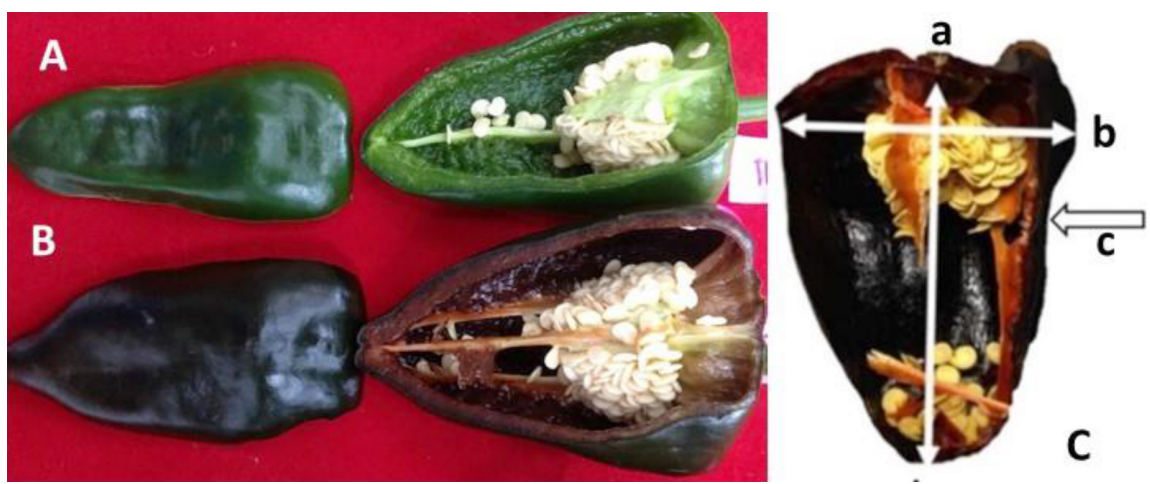

Figura 1. Fruto fresco de chile huacle: A) antes y B) después de madurez fisiológica. C) Fruto seco: a) ancho, b) largo y c) pericarpio. 
Tabla 2. Características físicas de frutos de chile huacle.

\begin{tabular}{ccccccc}
\hline $\begin{array}{c}\text { Tratamiento } \\
(\text { meq L }\end{array}$ & $\begin{array}{c}\text { Peso seco } \\
(\mathrm{g})\end{array}$ & $\begin{array}{c}\text { Largo } \\
(\mathrm{cm})\end{array}$ & $\begin{array}{c}\text { Ancho } \\
(\mathrm{cm})\end{array}$ & $\begin{array}{c}\text { Grosor de pericarpio } \\
(\mathrm{mm})\end{array}$ & $\begin{array}{c}\text { Número de } \\
\text { semillas }\end{array}$ & $\begin{array}{c}\text { Peso de semillas } \\
(\mathrm{g})\end{array}$ \\
\hline 20 & $5,56 \mathrm{a}$ & $7,10 \mathrm{a}$ & $4,21 \mathrm{a}$ & $0,31 \mathrm{a}$ & $203 \mathrm{a}$ & $1,25 \mathrm{a}$ \\
15 & $4,82 \mathrm{~b}$ & $6,75 \mathrm{a}$ & $3,80 \mathrm{~b}$ & $0,30 \mathrm{a}$ & $188 \mathrm{a}$ & $1,12 \mathrm{a}$ \\
10 & $4,48 \mathrm{~b}$ & $6,05 \mathrm{~b}$ & $3,74 \mathrm{~b}$ & $0,29 \mathrm{a}$ & $173 \mathrm{a}$ & $1,0 \mathrm{a}$ \\
\hline
\end{tabular}

Promedios con la misma letra en columnas son iguales estadísticamente (Tukey, 0,05).

Todas las correlaciones entre las variables físicas del fruto (Tabla 3 ) fueron altamente significativas $(\mathrm{P} \leq 0,01)$. Con $20 \mathrm{meq} \mathrm{L}^{-1}$ la correlación presentada en la interacción ancho con el número y peso de semillas fueron mayor a las otras dos concentraciones, debido probablemente a la mayor disponibilidad de nutrientes para la planta y a la mayor cantidad y peso de semillas. Al respecto, Copeland y McDonald (2001) mencionan que el aumento en el peso de las semillas es debido a la acumulación de nutrientes y agua, asociado con la rápida división y elongación celular, lo cual probablemente sucedió en este estudio. Asimismo, Thanopoulos et al. (2013) observaron que los frutos de chile pimiento con semillas fueron de mayor peso y tamaño en comparación a los frutos sin semillas.

\section{Características físicas de las semillas}

En el análisis de varianza de las variables de semillas (Tabla 4), las diferencias fueron altamente significativas $(\mathrm{P} \leq 0.01)$, lo cual implica que las características de la semilla variaron de acuerdo a la concentración de la solución Steiner.

Kraft et al. (2010) mencionan que el tamaño de la semilla es el principal criterio utilizado por agricultores de chiles en México para la selección de

Tabla 3. Correlaciones en características físicas de fruto del chile huacle.

\begin{tabular}{|c|c|c|c|}
\hline Variable & $20\left(\right.$ meq L $\left.^{-1}\right)$ & $15\left(\right.$ meq L $\left.\mathrm{L}^{-1}\right)$ & $10\left(\right.$ meq L $\left.^{-1}\right)$ \\
\hline $\mathrm{PSF} v s \mathrm{~L}$ & $0,700 * *$ & $0,693 * *$ & $0,780 * *$ \\
\hline PSF vs A & $0,877 * *$ & $0,625^{* *}$ & $0,711 * *$ \\
\hline $\mathrm{PSF} v s \mathrm{Ns}$ & $0,792 * *$ & $0,707 * *$ & $0,559 * *$ \\
\hline PSF $v s$ Ps & $0,793 * *$ & $0,707 * *$ & $0,546^{* *}$ \\
\hline $\mathrm{L} v s \mathrm{~A}$ & $0,573 * *$ & $0,564 * *$ & $0,672 * *$ \\
\hline A vs Ns & $0,851 * *$ & $0,627 * *$ & $0,738 * *$ \\
\hline A $v s$ Ps & $0,853^{* *}$ & $0,624 * *$ & $0,726^{* *}$ \\
\hline
\end{tabular}

$* *$ =Altamente significativo $(\mathrm{P} \leq 0,01) . \mathrm{PSF}=$ peso seco de fruto, $\mathrm{L}=$ largo, $\mathrm{A}=$ ancho, $\mathrm{Ns}=$ número de semillas, $\mathrm{Ps}=$ peso de semillas. la semilla que utilizarán en los nuevos ciclos. En la Tabla 5, se muestra que con 20 meq L $\mathrm{L}^{-1}$, los valores de las variables: área, largo y peso de 1,000 semillas fueron mayores con respecto a los otros tratamientos. En cuanto al tamaño de las semillas estas fueron de menor tamaño respecto a lo reportado por Bosland y Votava (2012), quienes mencionan que una semilla típica de Capsicum annuum tiene aproximadamente $1 \mathrm{~mm}$ de espesor, 5,3 $\mathrm{mm}$ de largo, 4,3 $\mathrm{mm}$ de ancho y una superficie de $33 \mathrm{~mm}^{2}$. Con respecto al peso de las 1,000 semillas, en todos los tratamientos fueron similares a los encontrados por Pablo et al. (2009) en diferentes colectas de chile de agua con fluctuación de 4,84 a 6,24 g por 1,000 semillas.

\section{Contenido de humedad}

Las semillas de los tres tratamientos presentaron en promedio $7 \%$ de humedad, valor similar a

Tabla 4. Cuadrados medios y significancia estadística de las variables de la semilla de chile huacle.

\begin{tabular}{lccc}
\hline Variable & Cuadrados Medios & Significancia & CV \\
\hline Área & 155,616 & $* *$ & 10,22 \\
Largo & 3,328 & $* *$ & 5,75 \\
Ancho & 3,211 & $* *$ & 3,92 \\
Peso & 0,003 & $* *$ & 3,3 \\
\hline
\end{tabular}

$\mathrm{CV}=$ Coeficiente de variación, $* *$ altamente significativo $(\mathrm{P} \leq 0,01)$.

Tabla 5. Tamaño y peso de semillas de chile huacle.

\begin{tabular}{ccccc}
\hline $\begin{array}{c}\text { Tratamiento } \\
\left(\text { meq L }^{-1}\right)\end{array}$ & $\begin{array}{c}\text { Área } \\
\left(\mathrm{mm}^{2}\right)\end{array}$ & $\begin{array}{c}\text { Largo } \\
(\mathrm{mm})\end{array}$ & $\begin{array}{c}\text { Ancho } \\
(\mathrm{mm})\end{array}$ & $\begin{array}{c}\text { Peso de } 1,000 \\
\text { semillas }(\mathrm{g})\end{array}$ \\
\hline 20 & $15,9 \mathrm{a}$ & $4,9 \mathrm{a}$ & $4,0 \mathrm{a}$ & $6,19 \mathrm{a}$ \\
15 & $15,5 \mathrm{~b}$ & $4,8 \mathrm{~b}$ & $4,0 \mathrm{a}$ & $5,99 \mathrm{~b}$ \\
10 & $14,7 \mathrm{c}$ & $4,7 \mathrm{c}$ & $3,9 \mathrm{~b}$ & $5,79 \mathrm{c}$ \\
\hline
\end{tabular}

Promedios con la misma letra en columnas son iguales estadísticamente (Tukey, 0,05). 
lo reportado en otros tipos de chiles (Capsicum annuит L.; Ayala-Villegas et al., 2014) y en jitomate (Licopersicum esculentum L.; Da Silva Almeida et al., 2014), porcentajes cercanos al rango óptimo (8 a 10\%) indicado por Reveles Hernández et al. (2013) para el almacenamiento de semillas de chiles.

\section{Viabilidad}

En los tres tratamientos, la prueba de cloruro de tetrazolio mostró un porcentaje promedio del 78 al $96 \%$ de viabilidad. La solución nutritiva de 20 meq L $\mathrm{L}^{-1}$, mostró mayor tonalidad en la tinción de cotiledones y embrión, factores considerados para semillas viables con alto vigor (Figura 2). Lo cual sucede al incrementarse la actividad de las deshidrogenasas durante la fase inicial de la germinación, al iniciar la liberación de iones hidrógeno que reducen la solución de tetrazolio y se sintetiza el formazán, éste compuesto es rojo y demuestra la presencia de células vivas en los tejidos seminales (Gutiérrez-Hernández et al., 2011).

\section{Germinación y envejecimiento acelerado}

En la comparación de medias Tukey se detectaron diferencias significativas $(P \leq 0.05)$ en las variables germinación, plúmula y radícula

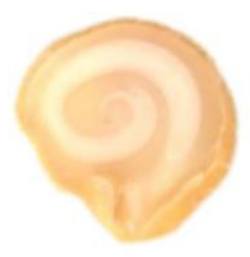

A

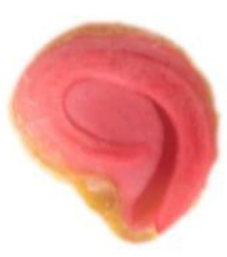

B

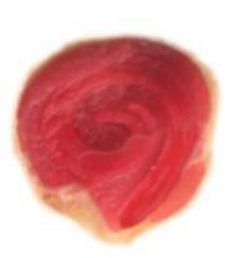

C
Figura 2. Viabilidad y vigor de las semillas de chile huacle tratadas con cloruro de tetrazolio: (A) no viable, (B) viable con vigor medio y $(\mathrm{C})$ viable con alto vigor.
(Tabla 6), características que variaron debido a la concentración solución Steiner. El mayor porcentaje de germinación fue con la solución Steiner $\left(20\right.$ meq $\left.\mathrm{L}^{-1}\right)$, debido también al mayor peso de las semillas (Tabla 5), es una correlación consistente con lo encontrado por Da TrindadeLessa et al. (2015) en semillas de Enterolobium contortisiliquum. Asimismo, el porcentaje de germinación en tdos los tratamientos fue mayor al $43 \%$ reportado en semillas de chile amashito (Capsicum annuum L. var. glabriusculum; González-Cortés et al., 2015) y similares al $81 \%$ de chile habanero (Garruña-Hernández et al., 2014) y con el $95 \%$ en chile pimiento (Kim et al., 2014) y la mayor longitud de radícula (Tabla 6) por efecto de la solución Steiner $\left(20 \mathrm{meq} \mathrm{L}^{-1}\right)$ fue similar a los 5,29 cm encontrado en semillas de chile de agua por Pablo et al. (2009).

Asimismo, al incrementar la concentración de solución Steiner, se redujo el porcentaje de germinación de 6 a $12 \%$ después de ser sometidas a envejecimiento acelerado (Tabla 6), debido probablemente a que el envejecimiento conduce a la disminución de la síntesis de ADN y al aumento en la degradación del mismo (Hussein et al., 2011). Sin embargo, aún con la reducción en el porcentaje de germinación después del envejecimiento acelerado y de acuerdo a González Rodríguez et al. (2014), las semillas de los tres tratamientos pueden considerarse con alto vigor porque la germinación fue del $80 \%$. Por otra parte, no hubo diferencias significativas en la longitud de la radícula ni en la germinación con el envejecimiento acelerado; en cambio, si lo hubo en las semillas no sometidas a envejecimiento (Tabla 6). Los resultados obtenidos son consistentes en cuanto a la reducción en el porcentaje de germinación del chile huacle después de haber sido sometidas al envejecimiento acelerado como en jitomate (Lycopersicum esculentum; da Silva Almeida et al., 2014).

Tabla 6. Porcentaje de germinación y características de plántulas de chile huacle.

\begin{tabular}{|c|c|c|c|c|c|c|}
\hline \multirow{2}{*}{ Tratamiento (meq L ${ }^{-1}$ ) } & \multicolumn{3}{|c|}{ Sin envejecimiento } & \multicolumn{3}{|c|}{ Con envejecimiento } \\
\hline & Germinación \% & Plúmula $(\mathrm{cm})$ & Radícula (cm) & Germinación \% & Plúmula $(\mathrm{cm})$ & Radícula $(\mathrm{cm})$ \\
\hline 20 & $96 \mathrm{a}$ & $2,54 \mathrm{a}$ & $5,02 \mathrm{a}$ & $84 \mathrm{a}$ & $2,64 \mathrm{a}$ & $1,52 \mathrm{a}$ \\
\hline 15 & $87 \mathrm{~b}$ & $2,65 \mathrm{a}$ & $4,05 \mathrm{~b}$ & $81 \mathrm{a}$ & $2,48 \mathrm{ab}$ & $1,09 \mathrm{a}$ \\
\hline 10 & $80 \mathrm{c}$ & $2,40 \mathrm{a}$ & $3,79 \mathrm{~b}$ & $80 \mathrm{a}$ & $2,27 \mathrm{~b}$ & $1,12 \mathrm{a}$ \\
\hline
\end{tabular}

Promedios con la misma letra en columnas son iguales estadísticamente (Tukey, 0,05). 


\section{Conclusiones}

Las plantas tratadas con la solución Steiner de $20 \mathrm{meq} \mathrm{L}^{-1}$ aplicada a plantas de chile huacle produjeron frutos con mayor peso seco $(5,56 \mathrm{~g})$, largo $(7,1 \mathrm{~cm}) \mathrm{y}$ ancho $(4,21 \mathrm{~cm})$. Asimismo, semillas obtenidas con la misma concentración aumentaron su calidad física y fisiológica mostrando un área de $15,9 \mathrm{~mm}^{2}$, largo de 4,9 mm, 6,19 $\mathrm{g}$ en el peso de 1,000 semillas, $96 \%$ de germinación y plántulas con la radícula de mayor tamaño $(5,02 \mathrm{~cm})$ respecto a los obtenidos con los tratamientos de 15 y 10 meq L $^{-1}$ de la solución Steiner.

Las semillas de todos los tratamientos presentaron $80 \%$ de germinación posterior al envejecimiento acelerado, considerando con ello a todas las semillas con alto vigor, característica deseable para su almacenamiento y preservación.

\section{Agradecimientos}

Al Instituto Politécnico Nacional, por el financiamiento otorgado al proyecto de chile huacle SIP 20180619. Al CONACyT por la beca otorgada para estudios de posgrado del estudiante. Dr. Serafín Cruz Izquierdo, por todas la facilidades brindadas al estudiante de posgrado durante su estancia de Investigación en el Colegio de Postgraduados.

\section{Literatura Citada}

Alejo-Santiago G., Luna-Esquivel, G., Sánchez-Hernández, R., Salcedo-Pérez, E., García-Paredes, J.D., Jiménez-Meza, V.M. 2015. Determination of the nitrogen requirement for habanero pepper (Capsicum chinense Jacq,) Revista Chapingo Serie Horticultura. 21(3): 215-227.

Ayala-Villegas, M.J., Ayala-Garay, O.J., Aguilar-Rincón, V.H., Corona-Torres, $\mathrm{T}$.

2014. Evolución de la calidad de semilla de Capsicum annuum L. durante su desarrollo en el fruto. Revista Fitotecnia Mexicana 37: 79-87.

Bosland, P.W., Votava, E.J.

2012. Peppers: Vegetable and Spice Capsicums (2nd ed). CABI, USA. 230 p.

Copeland, L.O., McDonald, M.B.

2001. Principles of Seed Science and Technology (4th ed.). Springer, USA. 467 p.

Cruz-Crespo, E., Can-Chulim, A., Bugarín-Montoya, R., Pineda-

Pineda, J., Flores-Canales, R. Juárez-López, P., Alejo-Santiago, G. 2014. Concentración nutrimental foliar y crecimiento de chile serrano en función de la solución nutritiva y el sustrato. Revista Fitotecnia Mexicana 37: 289-295.

Da Silva Almeida, A., Deuner, C., Terra Borges, C., Meneghello,

G.E., Madruga de Tunes, L., Amaral Villela, F.

2014. Accelerated aging in tomato seeds. American Journal of Plant Sciences 05: 1651-1656.

Da Trindade-Lessa, B.F., Nobre-de Almeida, J.P., Lobo-Pinheiro,

C., Melo-Gomes, F., Medeiros-Filho, S.

2015. Germinación y crecimiento de plántulas de Enterolobium contortisiliquum en función del peso de la semilla y las condiciones de temperatura y luz. Agrociencia 49: 315-327

Garruña-Hernández, R., Latournerie-Moreno, L., Ayala-Garay,

O., Santamaría, J.M., Pinzón-López, L.

2014. Acondicionamiento pre-siembra: una opción para incrementar la germinación de semillas de chile habanero (Capsicum chinense Jacq.). Agrociencia 48: 413-423.

González-Cortés, N., Jiménez Vera, R., Guerra Baños, E.C., Silos Espino, H., Payro de la Cruz, E.

2015. Germinación del chile amashito (Capsicum annuum L. var. Glabriusculum) en el sureste mexicano. Revista Mexicana de Ciencias Agrícolas 11: 2211-2218.
González Rodríguez, F., León Gómez, D., Borges Gómez, L., Pinzón López, L., Magaña Magaña, M., Sangines García R., Urrestarazu Gavilán, M.

2014. Envejecimiento acelerado sobre la calidad de semillas de maíz para producir germinados para forraje alternativo. Revista Mexicana de Ciencias Agrícolas 8:1487-1493.

Gutiérrez-Hernández, G.F., Vázquez-Ramos, J.M., GarcíaRamírez, E., Franco-Hernández, M.O., Arellano-Vázquez, J.L., Durán-Hernández, D.

2011. Efecto del envejecimiento artificial de semillas de maíces criollos azules en su germinación y huella genómica. Revista Fitotecnia Mexicana 34: 77-84.

Hussein, H.J., A.I. Shaheed y O.M. Yasser.

2011. Effect of accelerated aging conditions on viability of sunflower (Helianthus annus L.) seeds. Euphrates Journal of Agriculture Science 3: 1-9.

ISTA (International Seed Testing Association).

2012. International Rules for Seed Testing Zurich, Switzerland ISBN-13978-3-906549-68-2.

Izquierdo J., Granados-Ortiz, S.

2011. Producción artesanal de semillas de hortalizas para la huerta familiar. Manual Técnico. FAO. 98p.

Kim, G., Kim, G.H., Lohumi, S., Kang, J.S., Cho, B.K. 2014. Viability Physics and Technology 67: 214-221.

Kraft, K. H., Luna Ruiz, J.J., Gepts P.

2010. Different seed selection and conservation practices for fresh market and dried chile farmers in Aguascalientes, Mexico. Economic Botany 64: 318-328.

López-López, P., Rodríguez-Hernández, R., Bravo-Mosqueda, E. 2016. Impacto económico del chile huacle (Capsicum annuum L.) en el estado de Oaxaca. Revista Mexicana de Agronegocios 38: 317-328.

Pablo Carrillo, E., Mejía Contreras, J. A., Carballo Carballo, A., García de los Santos, G., Aguilar Rincón, V.H., Corona Torres T. 2009. Calidad de semillas en colectas de chile de agua (Capsicum annuum L.) de los valles centrales de Oaxaca, México. Agricultura Técnica en México 35: 257-266.

Ramalho do Rêgo E., Monteiro do Rêgo, M., Luiz Finger, F. 2016. Production and Breeding of Chilli Peppers. Springer international Publishing. $134 \mathrm{p}$. 
Reveles Hernández, M., Velásquez Valle, R., Reveles Torres L.R., Mena Covarrubias, J.

2013. Selección y conservación de semilla de chile: primer paso para una buena cosecha. Folleto Técnico. Número 51. Campo Experimental Zacatecas. CIRNOC-INIFAP. $43 \mathrm{p}$.

SAS (Statistical Analysis System).

2002. User's Guide, Volumes 1 and 2 SAS/ ETS 9. Institute Inc. Cary, NC, EUA. 2143 p.

Tapia-Vargas M., Larios-Guzmán, A., Días-Sánchez, D.D., Ramírez-Ojeda, G., Hernández-Pérez, A., Vidales-Fernández, I., Guillén-Andrade, $\mathrm{H}$.

2016. Producción hidropónica de chile habanero negro (Capsicum Chinense Jacq.). Revista Fitotecnia Mexicana 39(3): 241-245).
Thanopoulos, C., Bouranis, D., Passam H.C.

2013. Comparative development, maturation and ripening of seedless and seed-containing bell pepper fruits. Scientia Horticulturae 164: 573-577.

Valadez Sánchez Y.M., Olivares Sáenz, E., Vázquez Alvarado, R.E., Esparza-Rivera, J.R., Preciado- Rangel, P., Valdez- Cepeda, R.D., García Hernández, J.L.

2016. Calidad y concentración de capsaicinoides en genotipos de chiles Serrano (Capsicum annuum L.) producidos bajo fertilización orgánica. Revista Internacional de Botánica Experimental 85: 21-26.

Valentín-Miguel M. C., Castro-Brindis, R., Rodríguez-Pérez, J.E., Pérez-Grajales, M.

2013. Extracción de macronutrimentos en chile de agua (Capsicum annuит L.). Revista Chapingo Serie Horticultura 19 (4): 71-78. 
